\title{
A Variational Rod Model with a Singular Nonlocal Potential
}

\author{
Kathleen A. Hoffman and Thomas I. Seidman \\ Department of Mathematics and Statistics \\ University of Maryland Baltimore County \\ Baltimore, MD 21250, USA \\ (khoffman, seidman@math.umbc.edu)
}

\begin{abstract}
In the classic theory of elastic rods, two non-adjacent points along the rod may upon contact occupy the same physical space. We develop an elastic rod model with a pairwise repulsive potential such that if two non-adjacent points along the rod are close in physical space, there is an energy barrier that prevents contact. For adjacent pairs, the repulsive potential is negligible and the elastic rod is described by a classical elastic rod model. The framework for this model is developed to prove the existence of minimizers within each homotopy class, where the idea of topological homotopy of a curve is generalized to a framed curve, or an elastic rod. Finally, the first-order necessary conditions are derived.
\end{abstract}

\section{Introduction}

Although a focus of scientific study for centuries, much of the scientific literature on elastic rod theory has neglected the contact problem, formally permitting rods to have self-intersecting configurations: for 'almost straight' rods this neglect is nugatory while incorporating the contact forces is an analytically challenging and computationally expensive task.

Recently there has been increased interest in elastic rod theories which go beyond the classical theory and eliminate the possibility of self-penetration. Elastic rod theory is typically formulated in terms of an energy functional whose minima correspond to stable equilibria. Generally, such models fall into one of two categories: soft contact techniques by pairwise repulsive potentials, which are naturally singular, and the hard contact approach, in which a tubular neighborhood surrounds the centerline of the curve imposing a fixed positive thickness $\rho$. This paper falls into the first of these categories, developing an appropriate pairwise repulsive potential to model the impenetrability of the rod. This paper is concerned with the 
existence of minima of elastic rod energies using these pairwise repulsive potentials. Building on classical existence results (see, for example, $[1,2,3,4,6,8]$ ), we prove the existence of minima within each homotopy class while extending the traditional definition of homotopy of a curve to framed curves, or elastic rods. We also develop the consequent nonlocal equilibrium equations and discuss regularity.

The hard contact model is challenging because of the discontinuities arising at the boundary of the contact region. Schuricht [28, 29, 30, 19] and collaborators have used the Clarke derivative to overcome these analytical challenges, focusing on three dimensional elastic bodies. Gonzalez et al. [15, 16] developed the idea of a global radius of curvature. They consider the circumcircle radius as a function of three points on the curve, which they call the global radius of curvature function. The constraint on the radius of the centerline is then given by a prescribed positive lower bound on the global radius of curvature function as one varies among all possible triplets of distinct points along the curve (compare Lemma 2). Their formulation is an analytically tractable notion of 'hard contact' and has inspired a sequence of papers addressing various existence and regularity questions of this formulation $[7,31,36]$. Coleman and Swigon $[10,9]$ developed tests on the second-order variation to determine which equilibria correspond to minima for elastic rod models where opposing forces are introduced at points of self-contact, whereas van der Heijden et al. [37] focus on finding bifurcation diagrams that identify and classify "jump phenomena" at points of self-contact. Starostin [35] has investigated the computation of non-penetrating equilibria by assembling them from simple loop elements and exploiting the symmetries of the problem; in particular cases, the problem admits an analytic solution [34]. Delrow [11] investigates the validity of the hard contact assumption in an elastic model of DNA with Coulomb interactions. Strzelecki et al. [36] studied an energy functional that includes the elastic potential energy and a contact term intermediate between pairwise repulsive potentials and hard obstacle conditions; they further develop the conditions under which existence of minimizers is guaranteed within a given homotopy class.

Where most of the literature on contact of elastic rods has focused on the hard contact problem, it is interesting to note that knot theorists have created a significant literature using the soft contact approach. Knot theorists seek optimal knot representatives as energy minimizers within a given knot class [14, 33, 26, 21]. Existence and regularity results exist for the well-known O'Hara knot energy for inverse square repulsive potentials [26]; similar results in higher dimensions have been developed by [24]. The pairwise repulsive potentials considered by knot theorists can also be used to model soft contact of elastic rods; however the existence and regularity results will vary due to the elastic potential energy included in the energy functional 
of the elastic rods that is not present in knot energies.

In this paper, as noted above, we focus on developing a general soft contact model involving a pairwise repulsive energy that imposes an increasingly large energy barrier when two non-adjacent points along the rod become closer to each other in space. Our approach may be viewed as a penalty function approach to the hard contact problem, yet also can closely reflect the physics of the problem by choice of the energy penalty. Several physically motivated potential functions are plausible choices, such as Coulomb electrostatic repulsion, a Lennard-Jones potential, or the Debye-Huckle potential, but we will not make any specific selection.

The article is organized as follows. Section 2 contains a brief review of some geometric aspects of the theory of elastic rods, discusses geometric estimates for self-contact and defines rod homotopy. Section 3 contains the energy formulation in terms of the internal and external potentials, but also further classifies the internal energy as either local (classical) or non-local. Assumptions required for the existence theorem are presented and discussed in section 3. Well-posedness of the problem is discussed in section 4 for the total energy developed in section 3: an existence theorem is formulated and proved and continuous dependence on data is discussed. Section 5 derives the first-order necessary conditions. Section 6 considers existence in a setting, corresponding to coupling with temperature dependent material properties, in which the local internal energy function is not convex when expressed in terms of the strains and section 7 concludes with a discussion of the results obtained and some further considerations.

\section{Problem Formulation: Geometry}

This section considers only the geometry of the rod and is independent of the physics. The physical properties of the rod model enter only in connection with the energy functional and its constitutive functions and we defer our discussion of those issues to the next section. We have chosen to include here not only a geometric description of elastic rod theory, but also other topics which rely only on the geometry of the problem and not on the underlying physics. Examples include the idea of rod homotopies and a nonlocality inequality.

\subsection{The Cosserat Rod Description}

We wish to consider a (possibly) nonlinear elastic rod model that is geometrically exact for large deformations of inextensible and unshearable elastic rods. This is a special case of the special Cosserat theory of rods. A comprehensive discussion of 
the Cosserat theory can be found in [5] and we present here only a brief description of the geometrical ideas designed to set notation.

The configuration of a rod is here described by a centerline $s \mapsto \mathbf{r}(s)$ (which we write as a function of arc length $s$ along the rod, assuming units chosen so $0 \leq s \leq 1$ ) and a set $\mathbf{q}(s)=\left\{\mathbf{d}_{1}(s), \mathbf{d}_{2}(s), \mathbf{d}_{3}(s)\right\}$ of directors forming an orthonormal frame describing the orientation of the cross-section of the rod. [At this point we might introduce a tubular radius $\rho \geq 0$ for the rod, but this plays no role in the Cosserat description so the idealization $\rho=0$ is admissible for our theory.] We take $\mathbf{d}_{1}, \mathbf{d}_{2}$ as attached to the cross-section with $\mathbf{d}_{3}=\mathbf{d}_{1} \times \mathbf{d}_{2}$ so each $\mathbf{q}(s)$ is a $3 \times 3$ matrix in $S O(3)$. Without loss of generality we may assume our coordinate system has been chosen so

$$
\mathbf{q}(0)=\mathbf{I} \quad \mathbf{r}(0)=\mathbf{0} .
$$

For convenience we restrict our attention to inextensible and unshearable rods for which the third director $\mathbf{d}_{3}$ coincides with the tangent vector to the centerline:

$$
\mathbf{d}_{3}(s)=\mathbf{r}^{\prime}(s) \text {. }
$$

[Our results could be extended to include both shearable and extensible rods, but the assumption (2.2) substantially simplifies some of the arguments. This assumption further restricts the special Cosserat rod theory.]

The assumed orthonormality of the directors $\left\{\mathbf{d}_{i}(s)\right\}$ implies the existence of a (Darboux) vector $\mathbf{u}(s)$, definable by the relation

$$
\mathbf{d}_{i}^{\prime}(s)=\mathbf{u}(s) \times \mathbf{d}_{i}(s) \quad(i=1,2,3)
$$

and we then express $\mathbf{u}(s)$ in the local rod frame with coefficients $u_{i}$ so

$$
\begin{array}{ll}
u_{i}(s) \equiv \mathbf{u}(s) \cdot \mathbf{d}_{i}(s) & \text { giving } \mathbf{u}=\Sigma_{i} u_{i} \mathbf{d}_{i}=\mathbf{q U} \\
& \text { with } \mathbf{U}=u_{1} \mathbf{i}+u_{2} \mathbf{j}+u_{3} \mathbf{k}=\left(u_{1}, u_{2}, u_{3}\right)^{\top} \in \mathbb{R}^{3} .
\end{array}
$$

These $u_{i}$ are the strains in the model and we have the descriptive construction:

$$
\text { configuration } \longmapsto \text { strains: }(\mathbf{q}, \mathbf{r}) \mapsto \mathrm{U} \text {. }
$$

One advantage of the Cosserat theory is that this descriptor $\mathrm{U}(s)$ of the strains automatically has the appropriate frame indifference.

Now introduce $S: \mathrm{U} \mapsto[\mathrm{U} \times]$ as a skew matrix so

$$
S: \mathbb{R}^{3} \rightarrow \mathcal{M}=\mathcal{M}^{3 \times 3}:\left(\begin{array}{l}
u_{1} \\
u_{2} \\
u_{3}
\end{array}\right) \mapsto\left(\begin{array}{ccc}
0 & -u_{3} & u_{2} \\
u_{3} & 0 & -u_{1} \\
-u_{2} & u_{1} & 0
\end{array}\right)
$$


With $\mathrm{U}(\cdot)$ given, the definition $(2.3)$ of $\mathbf{u}$ then becomes the linear differential equation

$$
\mathbf{q}^{\prime}=\mathbf{q U} \times \mathbf{q}=\mathbf{q} S(\mathrm{U}) .
$$

The map: configuration $\longmapsto$ strains described above can be inverted by solving the pair of differential equations (2.2) and (2.3) along the rod:

$$
\mathbf{q}^{\prime}=\mathbf{q} S(\mathrm{U}) \quad \mathbf{r}^{\prime}=\mathbf{d}_{3}=\mathbf{q} \mathbf{k} \quad \text { for } 0 \leq s \leq 1
$$

with the initial conditions (2.1). We define the maps

$$
\begin{array}{ll}
\mathbf{Q}: \mathbf{U}(\cdot) \mapsto \mathbf{q}(\cdot): & L^{2}\left([0,1] \rightarrow \mathbb{R}^{3}\right) \rightarrow H^{1}([0,1] \rightarrow \mathcal{M}) \\
\mathbf{R}: \mathbf{q}(\cdot) \mapsto \mathbf{r}(\cdot): & H^{1}([0,1] \rightarrow \mathcal{X}) \rightarrow H^{2}\left([0,1] \rightarrow \mathbb{R}^{3}\right)
\end{array}
$$

by adjoining (2.1) to (2.6). The next section 2.2 contains a proof of the continuity and differentiability properties of these maps.

We conclude this subsection with the remark that equation (2.1) specifies the boundary conditions at the $s=0$ end of the rod, but boundary conditions at the $s=1$ end of the rod may further be imposed to complete the formulation and any such conditions on $\mathbf{q}, \mathbf{r}$ at $s=1$ (or elsewhere) remain as constraints on $\mathrm{U}(\cdot)$. There are numerous relevant possibilities at $s=1$ - for example:

- a free end with no further conditions imposed

- specification $\mathbf{r}(1)=\mathbf{r}_{1}$ with $\mathbf{r}_{1} \neq \mathbf{0}$ (either imposing conditions $\mathbf{q}(1)=q_{1}$, or leaving the rotation at $s=1$ free)

- periodicity conditions ( $\operatorname{so} \mathbf{r}(1)=\mathbf{0}, \mathbf{q}(1)=\mathbf{I})$ - in which case we use a mod 1 interpretation of $\Delta=s-\sigma$.

Except for details of the proof of Theorem 2, our analysis remains independent of any particular choice of boundary conditions at $s=1$, but these seem the most plausible alternatives.

\subsection{Properties of $\mathrm{Q}$ and $\mathrm{R}$}

In order to prove existence, and to develop conditions for optimality and regularity of the solutions, we establish properties of the maps $\mathbf{Q}$ and $\mathbf{R}$. Not commonly used in the theory of elastic rods, but convenient in this setting, is the Frobenius inner product and adjoints, which is described here. 
For matrices in the space $\mathcal{M}=\mathcal{M}^{3 \times 3}$ of $3 \times 3$ matrices we will use the Frobenius inner product $[M: N]_{F}=\operatorname{tr}\left(M^{\top} N\right)$ and the induced Frobenius norm (Euclidean norm on $\left.\mathbb{R}^{9} \sim \mathcal{M}^{3 \times 3}\right)$. It is not difficult to see from (2.5) that

$$
|\mathbf{q} S(\mathrm{U})|_{F}{ }^{2}=|S(\mathrm{U})|_{F}{ }^{2}=|\mathbf{u} \times \mathbf{q}|_{F}{ }^{2}=2|\mathbf{u}|^{2}=2|\mathrm{U}|^{2} .
$$

Thus, $S / \sqrt{2}$ and $\mathrm{U} \mapsto \mathbf{q} S(\mathrm{U}) / \sqrt{2}$ are isometries and each $\mathrm{U} \mapsto \mathbf{q}(s) S(\mathrm{U})$, which we may think of as a $3 \times 9$ matrix, is invertible from its range: the 3-dimensional tangent space at $\mathbf{q}(s)$ to $S O(3) \subset \mathcal{M}^{3 \times 3}$.

We will also need two Frobenius adjoints. For a map $A: \mathcal{M} \rightarrow \mathcal{M}$ we have the usual adjoint $A^{* F}$ so $\left[A^{* F}(L): M\right]_{F}=[L: A M]_{F}$; in particular, for right multiplication by $X \in \mathcal{M}$ ( i.e., $R(X): M \mapsto M X)$ the adjoint $[R(X)]^{* F}$ is the unique map: $\mathcal{M} \rightarrow \mathcal{M}$ such that

$$
\left[[R(X)]^{* F}(L): M\right]_{F}=[L: M X]_{F}
$$

[It seems inconveniently messy to work this out explicitly, e.g., as a matrix; for left multiplication, on the other hand, if $A: M \mapsto X M$, then $[A(X)]^{* F}: L \mapsto X^{\top} L$.] Second, for the linear map $S: \mathbb{R}^{3} \rightarrow \mathcal{M}$ we write $S^{* F}$ for the unique map: $\mathcal{M} \rightarrow \mathbb{R}^{3}$ such that

$$
S^{* F}(M) \cdot x=[M: S(x)]_{F} .
$$

Theorem 1. The map $\mathbf{Q}: \mathbf{U}(\cdot) \mapsto \mathbf{q}(\cdot)$ defined by (2.6) with boundary conditions (2.1) is continuous as in (2.7). We have $\left|\mathbf{q}^{\prime}(s)\right|_{F} \equiv \sqrt{2}|\mathbf{U}(s)|$ as in (2.8) and the map $\mathbf{Q}$ is continuously invertible from $H^{1}([0,1] \rightarrow S O(3))$ to $L^{2}\left([0,1] \rightarrow \mathbb{R}^{3}\right)$. Further, $\mathbf{Q}$ is Fréchet differentiable with

$$
\mathbf{Q}^{\prime}(\mathbf{U})=d \mathbf{Q} / d \mathbf{U}: L^{2}\left([0,1] \rightarrow \mathbb{R}^{3}\right) \rightarrow H^{1}([0,1] \rightarrow \mathcal{M}): \mathfrak{u} \mapsto \mathfrak{q}
$$

where $\mathfrak{q}=\mathbf{Q}^{\prime}(\mathbf{U}) \mathfrak{u}$ is the solution on $[0,1]$ of the linearized equation

$$
\mathfrak{q}^{\prime}=\mathfrak{q} S(\mathrm{U})+\mathfrak{q} S(\mathfrak{u}), \quad \mathfrak{q}(0)=\mathbf{0} .
$$

Finally, $\mathbf{Q}$ is continuous (and so necessarily compact) from the weak topology of $L^{2}\left([0,1] \rightarrow \mathbb{R}^{3}\right)$ to $C([0,1] \rightarrow \mathcal{M})$.

Proof. We begin with (2.8) so $\mathbf{Q}$ is as in (2.7). The continuity and differentiability of $\mathbf{Q}$ are standard for these spaces, using the Gronwall inequality for differences, etc., noting that $\mathbf{q}(s) \in S O(3)$ is uniformly bounded. [We may observe in passing that $\mathfrak{q}(s)$ is necessarily in the tangent space to $S O(3)$ at $\mathbf{q}(s)$ and thus will have the 
form $\mathfrak{q}(s)=\mathbf{q}(s) S(\mathfrak{z}(s))$.] Invertibility follows from the invertibility of $S$ from its range and the uniform invertibility of $\mathbf{q}(s)$.

The continuity from the weak topology is more interesting. Suppose $\mathbf{U}_{k} \rightarrow U$ in $L^{2}\left([0,1] \rightarrow \mathbb{R}^{3}\right)$ and let the functions $\mathbf{q}_{k}=\mathbf{Q}\left(\mathrm{U}_{k}\right)$ be obtained from $\mathrm{U}_{k}$ by (2.6) with (2.1); similarly, let $\mathbf{q}=\mathbf{Q}(\mathbf{U})$. Since $\left\{\left\|\mathbf{U}_{k}\right\|\right\}$ is bounded, so is $\left\{\left\|\mathbf{q}_{k}^{\prime}\right\|=\sqrt{2}\left\|\mathbf{U}_{k}\right\|\right\}$, whence we may extract a subsequence such that $\mathbf{q}_{k} \rightarrow \overline{\mathbf{q}}$ in $C\left([0,1] \rightarrow \mathcal{M}^{3 \times 3}\right)$ for some $\overline{\mathbf{q}}$. For each $s$ and any $M \in \mathcal{M}^{3 \times 3}$, the differential equation, integrated, gives

$$
\begin{aligned}
& \qquad \begin{aligned}
{\left[M: \mathbf{q}_{k}(s)-\mathbf{I}\right]_{F} } & =\int_{0}^{s}\left[M: \mathbf{q}_{k} S\left(\mathrm{U}_{k}(\sigma)\right)\right]_{F} d \sigma \\
& =\int_{0}^{1}\left\langle\mu_{k}^{s}(\sigma), \mathrm{U}_{k}(\sigma)\right\rangle d \sigma \quad \text { using }(2.9) \\
& =\left\langle\mu_{k}^{s}, \mathrm{U}_{k}\right\rangle \\
\text { where } \quad \mu_{k}^{s}(\sigma) & =\chi_{0, s}(\sigma) S^{* F}\left(\mathbf{q}_{k}(\sigma)^{\top} M\right) \in \mathbb{R}^{3} .
\end{aligned}
\end{aligned}
$$

Similarly, we set $\bar{\mu}^{s}(\sigma)=\chi_{0, s}(\sigma) S^{* F}\left(\overline{\mathbf{q}}(\sigma)^{\top} M\right)$ and note that, since $\mathbf{q}_{k} \rightarrow \overline{\mathbf{q}}$ uniformly on $[0,1]$, we have $\mu_{k}^{s} \rightarrow \bar{\mu}^{s}$ uniformly whence

$$
\left[M: \mathbf{q}_{k}(s)-\mathbf{I}\right]_{F}=\left\langle\mu_{k}^{s}, \mathbf{U}_{k}\right\rangle \longrightarrow\left\langle\bar{\mu}^{s}, \mathbf{U}\right\rangle=[M: \overline{\mathbf{q}}(s)-\mathbf{I}]_{F} .
$$

Since this holds for each $M$ and for each $s$, we see that $\overline{\mathbf{q}}$ satisfies pointwise

$$
\overline{\mathbf{q}}(s)=\mathbf{I}+\int_{0}^{s} \overline{\mathbf{q}}(\sigma) S(\mathrm{U}(\sigma)) d \sigma
$$

so $\overline{\mathbf{q}}$ is the unique solution $\mathbf{q}$ of the initial value problem of (2.6), (2.1). The uniqueness also ensures irrelevance of the possible subsequence extraction above so $\mathrm{U}_{k} \rightarrow \overline{\mathrm{U}}$ implies $\mathbf{q}_{k}=\mathbf{Q}\left(\mathrm{U}_{k}\right) \rightarrow \mathbf{Q}(\overline{\mathrm{U}})$ in the indicated sense.

Corollary 1 . The linear map $\mathbf{R}$ is clearly continuous from $L^{2}([0,1] \rightarrow \mathcal{M})$ to $H^{1}\left([0,1] \rightarrow \mathbb{R}^{3}\right)$; noting that $(2.3)$ and $(2.2)$ give $\mathbf{r}^{\prime \prime}=\mathbf{d}_{3}^{\prime}$, it is also continuous from $H^{1}([0,1] \rightarrow \mathcal{M})$ to $H^{2}\left([0,1] \rightarrow \mathbb{R}^{3}\right)$. The composed map $\mathbf{R} \circ \mathbf{Q}: \mathbf{U}(\cdot) \mapsto \mathbf{r}(\cdot)$ is continuous and compact to $C^{1}([0,1] \rightarrow \mathcal{M})$ from the weak topology of $L^{2}([0,1] \rightarrow$ $\left.\mathbb{R}^{3}\right)$.

We write $\mathbf{L}=d[\mathbf{R} \circ \mathbf{Q}] / d \mathbf{U}=\mathbf{R} \mathbf{Q}^{\prime}$, evaluated at some arbitrary $\mathbf{U} \in \mathbb{R}^{3}$ and proceed to compute the adjoint maps $\mathbf{R}^{*}$ and $\left[\mathbf{Q}^{\prime}\right]^{*}$ to obtain $\mathbf{L}^{*}=\left[\mathbf{Q}^{\prime}\right]^{*} \mathbf{R}^{*}$. For $\mathfrak{r}=\mathbf{L} \mathfrak{u}=\mathbf{R} \mathfrak{q}$, we have $d \mathfrak{r}(s) / d s=\mathfrak{q}(s) \mathbf{k}$ with $\mathfrak{q}$ satisfying (2.11) and we have $\mathfrak{r}^{\prime \prime}=[\mathbf{q} S(\mathrm{U})] \mathbf{k} \in L^{2}\left([0,1] \rightarrow \mathbb{R}^{3}\right)$ so $\mathbf{L u}=\mathfrak{r} \in H^{2}\left([0,1] \rightarrow \mathbb{R}^{3}\right)$. By duality we 
immediately get $\mathbf{L}^{*} v$ in $L^{2}\left([0,1] \rightarrow \mathbb{R}^{3}\right)$ for any $v$ in $H^{-2}\left([0,1] \rightarrow \mathbb{R}^{3}\right)$ but we will want to consider $v \in L^{2}\left([0,1] \rightarrow \mathbb{R}^{3}\right)$. One easily verifies that

$$
\begin{aligned}
& \langle v, \mathfrak{r}\rangle=\int_{0}^{1} v \cdot \mathfrak{q}^{\prime} d s=\int_{0}^{1} w \cdot \mathfrak{q} d s=\int_{0}^{1}[\mathbf{w}: \mathfrak{q}]_{F} d s \\
& \text { where } \quad w(s)=\int_{s}^{1} v \quad \mathbf{w}=(0,0, w) \text {. }
\end{aligned}
$$

We then have the following.

Lemma 1. The adjoint operator $\mathbf{L}^{*}$ is given by

$$
\left[\mathbf{L}^{*} v\right](s)=S^{* F}\left[\mathbf{q}^{\top}(s) \Theta(s)\right]
$$

where the $\mathcal{M}$-valued function $\Theta$ is obtained from $v$, using (2.12), as the solution of

$$
-\Theta^{\prime}=[R(S(\mathrm{U}(s)))]^{* F} \Theta+\mathbf{w}(s) \quad \Theta(1)=\mathbf{0} .
$$

For $v \in H^{-1}\left([0,1] \rightarrow \mathbb{R}^{3}\right)$, we then have $\mathbf{L}^{*} v \in H^{1}\left([0,1] \rightarrow \mathbb{R}^{3}\right) \subset C\left([0,1] \rightarrow \mathbb{R}^{3}\right)$.

Proof. We have

$$
\begin{aligned}
\langle v, \mathbf{L U}\rangle & =\int_{0}^{1}[\mathbf{w}: \mathfrak{q}]_{F} d s \\
& =\int_{0}^{1}\left[-\Theta^{\prime}-(A S(\mathbf{U}))^{* F} \Theta: \mathfrak{q}\right]_{F} d s \\
& =\int_{0}^{1}\left[\Theta: \mathfrak{q}^{\prime}\right]_{F} d s-\int_{0}^{1}\left[(A S(\mathbf{U}))^{* F} \Theta: \mathfrak{q}\right]_{F} d s \\
& =\int_{0}^{1}[\Theta: \mathfrak{q} S(\mathbf{U})+\mathbf{q} S(\mathfrak{u})]_{F} d s-\int_{0}^{1}\left[(A S(\mathbf{U}))^{* F} \Theta: \mathfrak{q}\right]_{F} d s \\
& =\int_{0}^{1}\left[\mathbf{q}^{\top} \Theta: S(\mathfrak{u})\right]_{F} d s=\int_{0}^{1} S^{* F}\left(\mathbf{q}^{\top} \Theta\right) \cdot \mathfrak{u} d s,
\end{aligned}
$$

which gives (2.13). For $v \in H^{-1}\left([0,1] \rightarrow \mathbb{R}^{3}\right)$, we have $\mathbf{w} \in L^{2}([0,1] \rightarrow \mathcal{M})$; since we know $\mathrm{U} \in L^{2}\left([0,1] \rightarrow \mathbb{R}^{3}\right)$, we also have $[R(S(\mathrm{U}))]^{* F} \in L^{2}([0,1] \rightarrow \mathcal{L}(\mathcal{M}))$ whence the solution $\Theta$ of $(2.14)$ is in $H^{1}([0,1] \rightarrow \mathcal{M})$. Since we also know that $\mathbf{q}=\mathbf{Q}(\mathrm{U}) \in H^{1}$ for $\mathrm{U} \in L^{2}$, it follows that the product $\mathbf{q}^{\top} \Theta$ is in $H^{1}$ whence $S^{* F}\left(\mathbf{q}^{\top} \Theta\right)=L^{*} v$ is in $H^{1}$ as asserted. 


\subsection{A Nonlocality Inequality}

Crucial to our approach will be an ability to distinguish effectively between parametric distances $\Delta$ (along the rod) and physical distances $D$ between elements:

$$
\Delta=|s-\sigma|, \quad D=D(s, \sigma)=|\mathbf{r}(s)-\mathbf{r}(\sigma)|
$$

(necessarily with $D \leq \Delta$ since we have required inextensibility so $\left|\mathbf{r}^{\prime}\right| \equiv 1$ ).

Lemma 2. For any $s, \sigma \in[0,1]$ we have

$$
\Delta \geq|\mathbf{r}(\sigma)-\mathbf{r}(s)| \geq\left(1-\sqrt{\Delta / 3}\left\|\mathbf{r}^{\prime \prime}\right\|\right) \Delta
$$

Proof. Without loss of generality, we assume $\sigma>s$ so $\Delta=\sigma-s>0$. To see (2.15), one need only note that $\Delta \geq D$ by inextensibility and

$$
\begin{aligned}
\mathbf{r}(\sigma)-\left[\mathbf{r}(s)+\Delta \mathbf{r}^{\prime}(s)\right] & =\int_{0}^{\Delta}(\Delta-\hat{s}) \mathbf{r}^{\prime \prime}(\hat{s}) d \hat{s} \quad \text { with } \\
\left|\int_{0}^{\Delta}(\Delta-\hat{s}) \mathbf{r}^{\prime \prime}(\hat{s}) d \hat{s}\right| & \leq\left[\int_{0}^{\Delta}(\Delta-\hat{s})^{2} d \hat{s}\right]^{1 / 2}\left\|\mathbf{r}^{\prime \prime}\right\|=\sqrt{\Delta^{3} / 3}\left\|\mathbf{r}^{\prime \prime}\right\|
\end{aligned}
$$

so $|D| \geq\left|\Delta \mathbf{r}^{\prime}\right|-\left|\int_{0}^{\Delta}(\Delta-\hat{s})^{2} \mathbf{r}^{\prime \prime}\right|$, giving (2.15).

With $\mathrm{U}$ bounded in $L^{2}\left([0,1] \rightarrow \mathbb{R}^{3}\right)$, this means that there is a well-defined gap between the case in which elements are physically close $(D \ll 1)$ just because they are very close along the rod (local propinquity) and those possible cases in which elements might be physically close in space despite having a significant parametric separation. With a somewhat arbitrary choice of $0<\vartheta<1$, we define this nonlocal propinquity more precisely by

$$
D \leq \vartheta \Delta
$$

and then note, as a consequence of Lemma 2, that (2.17) is only possible subject to a lower bound $\Delta_{1}>0$ on the parametric distance:

$$
D \leq \vartheta \Delta \quad \Longrightarrow \quad \Delta=|s-\sigma| \geq \Delta_{1}=\frac{3(1-\vartheta)^{2}}{\left\|\mathbf{r}^{\prime \prime}\right\|^{2}}
$$

It is then possible to define, as a functional on $\mathrm{U}(\cdot)$,

$$
D_{*}=\min \{D(s, \sigma): D \leq \vartheta \Delta\},
$$


somewhat arbitrarily setting $D_{*}=1$ if, as in the classical setting, there is no nonlocal interaction and this set is empty; otherwise, note that $\left\{(s, \sigma) \in[0,1]^{2}:|s-\sigma| \geq \Delta_{1}\right\}$ is compact so the minimum in (2.19) is attained.

For expository simplicity, we will be idealizing the cross-sectional radius as $\rho=0$ so $D_{*}=0$ means self-intersection/interpenetration. We refer to $D_{*}$ as the margin of separation or clearance, when this is positive.

At this point, we can define an admissible triple.

Definition 1. An admissible triple $[\mathrm{U}, \mathbf{q}, \mathbf{r}]$ satisfies

a. $\mathbf{U} \in L^{2}\left([0,1] \rightarrow \mathbb{R}^{3}\right)$ with $\mathbf{q}=\mathbf{Q}(\mathbf{U}), \mathbf{r}=\mathbf{R}(\mathbf{q})$ so $\mathbf{q} \in H^{1}\left([0,1] \rightarrow \mathcal{M}^{3 \times 3}\right)$ and $\mathbf{r} \in H^{2}\left([0,1] \rightarrow \mathbb{R}^{3}\right)$

b. any specified boundary conditions at $s=1$ are satisfied

c. $D_{*}>0$ so $\mathbf{r}$ has no self-intersection.

We also define convergence of a sequence of admissible triples.

Definition 2. A sequence of admissible triples converges $\left[\mathrm{U}_{k}, \mathbf{q}_{k}, \mathbf{r}_{k}\right] \rightarrow\left[\mathrm{U}_{\infty}, \mathbf{q}_{\infty}, \mathbf{r}_{\infty}\right]$ provided

a. $\mathbf{U}_{k} \rightarrow \mathrm{U}_{\infty}$, so $\mathbf{q}_{k} \rightarrow \mathbf{q}_{\infty}$ uniformly and $\mathbf{r}_{k} \rightarrow \mathbf{r}_{\infty}$ in $C^{1}\left([0,1] \rightarrow \mathbb{R}^{3}\right)$

b. there is a uniform positive lower bound on the margins of separation.

Note that this definition implies admissibility of the limit triple.

\subsection{Rod Homotopy}

In this subsection, we develop the idea of a rod homotopy, noting that the classical definition of a homotopy from one curve, for instance the centerline $\mathbf{r}$ of an elastic rod, to another is not adequate to define a homotopy between two elastic rod configurations because for rods we also want the directors and the strains to be considered. Thus, emphasizing (2.6), we define a rod homotopy as follows:

Definition 3. A rod homotopy joining $[\overline{\mathbf{U}}, \overline{\mathbf{q}}, \overline{\mathbf{r}}]$ to $[\hat{\mathbf{U}}, \hat{\mathbf{q}}, \hat{\mathbf{r}}]$ is a map: $t \mapsto \mathbf{q}(\cdot, t)$ inducing a map: $t \mapsto[\mathrm{U}(\cdot, t), \mathbf{q}(\cdot, t), \mathbf{r}(\cdot, t)]$ such that:

a. each $[\mathrm{U}(\cdot, t), \mathbf{q}(\cdot, t), \mathbf{r}(\cdot, t)]$ is an admissible triple, as in Definition 1

b. the map $t \mapsto \mathbf{q}(\cdot, t)$ is continuous from $[0,1]$ to $C([0,1] \rightarrow S O(3))$ 
c. $\mathbf{q}(\cdot, 0)=\overline{\mathbf{q}}(\cdot)$ and $\mathbf{q}(\cdot, 1)=\hat{\mathbf{q}}(\cdot)$

d. $\mathbf{q}(\cdot, t)$ is bounded (uniformly in $t$ ) in $H^{1}([0,1] \rightarrow S O(3)$ ); equivalently, $\mathrm{U}(\cdot, t)$ is uniformly bounded in $L^{2}\left([0,1] \rightarrow \mathbb{R}^{3}\right)$

e. we require that $D_{*}>0$ through the homotopy (uniformly in $t$ ) so $\mathbf{r}(\cdot, t)$ has no self-intersections and there is a uniform lower bound on the clearance.

With this definition, we can state the following theorem.

Theorem 2. Let $[\overline{\mathbf{U}}, \overline{\mathbf{q}}, \overline{\mathbf{r}}]$ be a specified admissible triple. Then there is a neighborhood $\mathcal{N}$ of $\overline{\mathbf{q}}(\cdot)$ in $C([0,1] \rightarrow S O(3))$ such that any $[\hat{\mathbf{U}}, \hat{\mathbf{q}}, \hat{\mathbf{r}}]$ with $\hat{\mathbf{q}} \in \mathcal{N}$ is rod homotopic to $[\overline{\mathrm{U}}, \overline{\mathbf{q}}, \overline{\mathbf{r}}]$.

Proof. Due to the separate treatment of various boundary conditions which might be imposed at $s=1$, the proof is long so we defer it to $\S 8.1$.

Corollary 2. Each rod homotopy class of admissible triples is closed with respect to the topology of Definition 3.

Proof. Suppose $\left[\mathrm{U}_{k}, \mathbf{q}_{k}, \mathbf{r}_{k}\right] \rightarrow[\overline{\mathbf{U}}, \overline{\mathbf{q}}, \overline{\mathbf{r}}]$ with each $\left[\mathrm{U}_{k}, \mathbf{q}_{k}, \mathbf{r}_{k}\right]$ an admissible triple in the same rod homotopy class. Convergence ensures, for each large enough $k$, that $\mathbf{q}_{k}$ will be in the neighborhood $\mathcal{N}$ of $\overline{\mathbf{q}}$ given by Theorem 2. The theorem then applies with $[\hat{\mathbf{U}}, \hat{\mathbf{q}}, \hat{\mathbf{r}}]=\left[\mathrm{U}_{k}, \mathbf{q}_{k}, \mathbf{r}_{k}\right]$ to show that the limit is also in the class.

\section{Problem Formulation: Energy}

As in classical continuum mechanics, we consider only short-range interactions acting over short distances, say, comparable to the length scale of the rod cross section. Unlike the classical formulation, which only takes into account interactions involving pairs of elements with $s, \sigma$ close to each other along the rod, we now include also any short-range nonlocal interactions involving pairs of elements with $s, \sigma$ far apart along the rod, but with $\mathbf{r}(s), \mathbf{r}(\sigma)$ close to each other physically. [In this formulation, as is consistent with the Cosserat rod model, we are requiring that these elements remain attached to their relative positions along the rod - excluding, for example, the direct consideration of a conductive rod in which binary interactions involve charges that are then forced to re-adjust their distribution along the rod.]

Fairly standard hypotheses on the classical component of the internal energy will provide a bound on $\left\|\mathbf{r}^{\prime \prime}\right\|$ and then Lemma 2 shows that we need not consider any intermediate scales. 
Our approach to these distinct situations will be to use the integral formulation:

$$
\int_{0}^{1} \int_{0}^{1} \mathfrak{g}(s, \sigma, \mathbf{r}(s), \mathbf{r}(\sigma)) d s d \sigma
$$

integrating the contributions of binary interactions between pairs of differential elements of the rod, $d s$ with $d \sigma$, in treating nonlocal interactions.

The local internal potential energy contribution should be the part of the energy cost due to local deformation of the rod when one would deform to $U$ from an unstressed reference configuration specified by $\hat{U}(s)$. [For a naturally straight rod, the unstressed configuration function would be $\hat{U} \equiv 0$. Our formulation is general enough to include $\hat{\mathrm{U}}(s) \neq 0$ as well as naturally anisotropic and non-uniform elastic rods.]

For very small $|s-\sigma|$ it is convenient to expess the deformation in terms of the difference quotient $[\mathbf{r}(s)-\mathbf{r}(\sigma))] /[s-\sigma]$ and go to the limit as an approximation so $\mathfrak{g}(s, \sigma, \mathbf{r}(s), \mathbf{r}(\sigma))$ becomes written in terms of $\mathbf{r}^{\prime}$. Phenomenologically, this local internal potential energy contribution $\mathcal{W}$ will be the standard elastic energy, given as usual by integrating a (material-dependent) strain energy density function:

$$
\mathcal{W}=\int_{0}^{1} W(s, \mathrm{U}(s)) d s .
$$

Note that $W(s, \cdot)$ at each point along the rod is here a function only of the strain $\mathrm{U}$, although perhaps depending on the material properties associated with that point. [Later, in subsection 6, we will consider a possibly interesting generalization of this.] Thus, we view the internal energy of the rod as the sum of two distinct pieces: the classical local potential energy $\mathcal{W}$ and a specifically nonlocal potential energy $\mathcal{G}$.

Since the forces are short-range, there can be a nonlocal contribution to the total internal potential energy only when distinct segments of the elastic rod have sufficiently small separation that the rod almost intersects itself. In this case, the classical interpretation of the internal forces as an appropriate derivative of the energy function is no longer valid (since $s \gg \sigma$ ). When the margin of separation of the rod is large as in the classical analysis, the contribution of the nonlocal portion of the internal potential energy becomes negligible and the total internal potential energy then properly given entirely by the classical formulation.

The idea of including nonlocal binary interactions in the potential energy of a continuum model is not new and has been investigated in solid mechanics $[23,27]$. Our formulation is similar in spirit to the peridynamic formulation proposed by Silling [32], which includes nonlocal binary terms over a length scale called a horizon. In contrast to the peridynamic formulation which includes long-range forces, the 
binary interactions in our model involve only short-range forces. It has been shown in the peridynamic literature that in the limit as the horizon goes to zero (i.e., in the limit of short-range local interactions), the peridynamic model converges to the classical continuum elastic model $[13,25]$; We take this as justifying our use of the classical elastic potential for purely local interaction.

\subsection{Nonlocal Internal Potential Energy}

We assume, for simplicity, that the nonlocal contribution to the internal potential energy depends only on the physical distance $D=D(s, \sigma)=|\mathbf{r}(s)-\mathbf{r}(\sigma)|$ between pairs of non-adjacent points along the elastic rod. In some sense, the integral in (3.2) is replacing the integration in (3.1) near the diagonal of $[0,1] \times[0,1]$ without requiring us to model truly local interactions in that binary form; compare $[13,25]$. Thus the nonlocal internal energy takes the form:

$$
\mathcal{G}=\int_{0}^{1} \int_{0}^{1} G(s, \sigma, D(s, \sigma)) d s d \sigma,
$$

where the constitutive function $G:[0,1]^{2} \times \mathbb{R}_{+} \rightarrow\left[-\beta_{G}, \infty\right]$ is lower semi-continuous with respect to $D$. Note that we are allowing negative values (bounded below by $\left.-\beta_{G}<0\right)$ for the constitutive function $G$ since we do not wish to exclude consideration of, e.g., the Lennard-Jones potential. We are also allowing the value $+\infty$ for $G$, see subsection 3.2.

\subsection{Soft Contact and a Clearance Inequality}

For $\mathcal{G}$ to be a realistic physical model which prohibits self-penetration of the elastic rod, we must ensure that with finite energy the rod cannot self-intersect. [For a homotopy or a dynamic model we similarly want it unable to pass through itself; compare Definition 4.] Thus, $\mathcal{G}$ should provide an infinite energy barrier as $D \rightarrow 0+$. We are primarily concerned with soft contact models, characterized by the imposition of a condition on the constitutive function $G$ under which an upper bound on the energy implies a positive lower bound on the margin of clearance for the physical separation: very near contact requires very large energy. To this end, we introduce the nonincreasing function

$$
\gamma(r)=\gamma(r ; G)=\inf \{G(s, \sigma, D): 0<D \leq r,|s-\sigma|>D / \vartheta\}
$$

and ask, as a condition on $G$, that $\gamma(\cdot ; G)$ satisfies

$$
\int_{0+}^{a} r \gamma(r) d r=+\infty
$$


Lemma 3. Let $G$ be such that (3.5) holds. Then there is a positive function $\delta_{*}$ such that $\mathcal{G}<c$ implies $D_{*} \geq \delta_{*}(c)>0$, i.e., for all nonlocal pairs $(s, \sigma)$ one would have a positive clearance: $|\mathbf{r}(s)-\mathbf{r}(\sigma)| \geq \delta_{*}(c)>0$.

We actually work with the inverse function $\delta \mapsto c_{*}(\delta)$, showing that if nonlocal segments of the rod were to approach each other to give $D \leq \delta$, then we must have $\mathcal{G} \geq c_{*}(\delta)$ with $c_{*}(\delta) \rightarrow \infty$ as $\delta \rightarrow 0+$ so a bound $\mathcal{G} \leq c$ provides a positive lower bound $\delta(c)$ on the clearance between the rod segments.

Proof. Suppose $0 \leq D_{*}=|\mathbf{r}(\alpha)-\mathbf{r}(\beta)| \leq \delta$ with $(\alpha, \beta)$ nonlocal. We assume, for convenience, that $(\alpha+s, \beta+\sigma)$ is also nonlocal for $r=\sqrt{s^{2}+\sigma^{2}} \leq a$ and note that $|\mathbf{r}(\alpha+s)-\mathbf{r}(\alpha)| \leq|s|,|\mathbf{r}(\beta+\sigma)-\mathbf{r}(\beta)| \leq|\sigma|$ so $D(\alpha+s, \beta+\sigma) \leq(\delta+\sqrt{2} r)$ and $G(\alpha+s, \beta+\sigma, D) \geq \gamma(\delta+\sqrt{2} r)$. Hence,

$$
\begin{aligned}
\mathcal{G} & \geq \iint_{0<r<a} G(\alpha+s, \beta+\sigma, D) d s d \sigma \\
& \geq \int_{0}^{2 \pi} \int_{0}^{a} \gamma(\delta+\sqrt{2} r) r d r d \theta=\pi \int_{0+}^{a / \sqrt{2}} \gamma(\delta+\hat{r}) \hat{r} d \hat{r} .
\end{aligned}
$$

Now the condition (3.5) implies that this last integral goes monotonically to $+\infty$ as $\delta \rightarrow 0+$, which is exactly sufficient to obtain the desired conclusion.

Somewhat complementarily to (3.5), we will also ask that

$$
G(s, \sigma, D)=0 \quad \text { for } D \geq D_{0}>0
$$

to emphasize that we are concerned only with short-range forces here, where, as with $\theta$, the cutoff $D_{0} \ll 1$ is selected somewhat arbitrarily. With a concern for Lemma 2, (3.6) serves to make a convenient separation between our treatments of local and nonlocal interactions. The precise nature of this cutoff is not really significant for our model, but of course any specific choice of $D_{0}$ means a possible neglect of some real interaction so the model may lose contact with reality at very high energy levels.

Note that the condition (3.5) holds for each of the potentials we have mentioned - Coulomb, Lennard-Jones, and Debye-Huckle. The complementary condition (3.6) does not hold automatically for these empirical potentials, but they already become quite small when not very far from their singularity so this makes only a minor change and we can easily enforce it, e.g., multiplying by a smooth cutoff function which is identically 1 for very small $D$, decreasing to be 0 for $D \geq D_{0}$. 
We remark that one might characterize a hard contact model from this perspective as requiring

$$
\rho>0, \quad G(s, \sigma, D) \begin{cases}=+\infty & \text { for } D<\rho, \\ \leq C<\infty & \text { for } D \geq \rho .\end{cases}
$$

\subsection{Total Energy}

The total energy functional consists of the internal potential energy (which we have split into a sum of local and nonlocal terms) and any external potential energy in the system - here assumed to have the form

$$
\mathcal{F}[\mathbf{r}]=\int_{0}^{1} F(s, \mathbf{r}(s)) d s
$$

where, for convenience, we have implicitly assumed some symmetry of the rod about its centerline in taking each $F(s, \cdot)$ to depend only on $\mathbf{r}(s)$ and not also on $\mathbf{q}(s)$.

As noted earlier, the local internal potential energy contribution is given by (3.2), integrating a (material-dependent) strain energy density function $W$. At this point, our only hypothesis regarding the constitutive function $W$ is a coercivity condition: $\|\mathrm{U}\| \rightarrow \infty \Longrightarrow \mathcal{W} \rightarrow \infty$ so a bound on $\mathcal{W}$ then bounds $\left\|\mathbf{r}^{\prime \prime}\right\|$. Sufficient for this coercivity condition would be a requirement that

$$
W(s, \mathrm{U}) \geq a|\mathrm{U}|-b
$$

for some positive constants $a, b$.

Combining the local, nonlocal and external contributions, the total energy $\mathcal{E}$ of the elastic rod is

$$
\mathcal{E}=\begin{array}{cccc}
\mathcal{W}[\mathbf{u}] & + & \mathcal{G}[\mathbf{r}] & \mathcal{F}[\mathbf{r}] \\
\int_{0}^{1}[W(s, \mathrm{U}(s)) & +\int_{0}^{1} G(s, \sigma,|\mathbf{r}(\sigma)-\mathbf{r}(s)|) d \sigma & +F(s, \mathbf{r}(s))] d s
\end{array}
$$

One may, of course, write $\mathcal{E}=\mathcal{E}[\mathrm{U}]$ since $\mathbf{r}=\mathbf{R Q}(\mathrm{U})$. The form (3.10) differs from the standard form precisely by its inclusion of the nonlocal term $\mathcal{G}$.

We impose the following assumptions on the constitutive functions $W, F, G$. 
(H)

a. Each of the functions $W, G, F$ is lower semicontinuous.

b. $W:[0,1] \times \mathbb{R}^{3} \longrightarrow\left[-\beta_{W}, \infty\right]$ is convex in its second variable and satisfies the coercivity condition (3.9)

c. $G:[0,1]^{2} \times \mathbb{R}_{+} \longrightarrow\left[-\beta_{G}, \infty\right]$ satisfies (3.5) and (3.6).

d. $F:[0,1] \times \mathbb{R}^{3} \longrightarrow \mathbb{R}$ is bounded below on bounded sets.

Lemma 4. If the constitutive functions $W, F, G$ satisfy the hypotheses $(\mathbf{H})$, then the energy functionals $\mathcal{W}, \mathcal{F}, \mathcal{G}$ satisfy

$(\mathcal{H})$

a. $\mathcal{W}: L^{2}\left([0,1] \rightarrow \mathbb{R}^{3}\right) \rightarrow\left[-\beta_{W}, \infty\right]$ is coercive and is lower semicontinuous from the weak topology

b. $\mathcal{G}: C\left([0,1] \rightarrow \mathbb{R}^{3}\right) \rightarrow\left[-\beta_{G}, \infty\right]$ is lower semicontinuous.

c. $\mathcal{F}: C\left([0,1] \rightarrow \mathbb{R}^{3}\right) \rightarrow\left[\beta_{F}, \infty\right]$ is lower semicontinuous.

Proof. It is standard that the properties $(\mathrm{a}, \mathrm{b}, \mathrm{c})$ in $(\mathcal{H})$ follow from the lower semicontinuity and, respectively, the corresponding properties (b,c,d) in $(\mathbf{H})$ for each of the constitutive functions.

If we strengthen these hypotheses slightly, additionally requiring that
a. $\mathcal{W}[\mathrm{U}]$ is bounded if $\|\mathrm{U}\|$ is bounded in $L^{2}\left([0,1] \rightarrow \mathbb{R}^{3}\right)$

b. $\mathcal{G}$ is continuous near each $\mathbf{r}$ where $\mathcal{G}[\mathbf{r}]<\infty$

c. $\mathcal{F}$ is continuous

then we can introduce a somewhat stronger homotopy notion:

Definition 4. A strong rod homotopy is a rod homotopy: $t \mapsto[\mathrm{U}(\cdot, t), \mathbf{q}(\cdot, t), \mathbf{r}(\cdot, t)]$ for which the condition $(d$.$) of Definition 3$ is replaced by the requirement that the total energy $\mathcal{E}[\mathrm{U}(\cdot, t)]$ should be bounded uniformly in $t \in[0,1]$.

Theorem 3. Assume $(\mathcal{H}),\left(\mathcal{H}^{\prime}\right)$ and let $[\overline{\mathbf{U}}, \overline{\mathbf{q}}, \overline{\mathbf{r}}]$ be a specified admissible triple. Then there is a neighborhood $\mathcal{N}$ of $\overline{\mathbf{q}}(\cdot)$ in $C([0,1] \rightarrow S O(3))$ such that any $[\hat{\mathbf{U}}, \hat{\mathbf{q}}, \hat{\mathbf{r}}]$ with $\overline{\mathbf{q}} \in \mathcal{N}$ is strongly rod homotopic to $[\overline{\mathbf{U}}, \overline{\mathbf{q}}, \overline{\mathbf{r}}]$. 
Proof. The proof of Theorem 2 guarantees that $\mathbf{q} \in H^{1}([0,1] \rightarrow S O(3))$, which implies that $\mathbf{q}^{\prime}$, and hence $U$ is bounded in $L^{2}\left([0,1] \rightarrow \mathbb{R}^{3}\right)(\mathrm{cf}$. Theorem 1 and (2.8)). Hypothesis (a.) in $\left(\mathcal{H}^{\prime}\right)$ guarantees that $\mathcal{W}[\mathrm{U}]$ is uniformly bounded. Condition (e.) in Definition 3, which was verified in the proof of Theorem 2, guarantees that $\mathcal{G}$ is bounded in $C\left([0,1] \rightarrow \mathbb{R}^{3}\right)$. Finally, $F$, and hence $\mathcal{F}$, is bounded by hypothesis.

\section{Well-Posedness}

Stable mechanical equilibria are obtained as (local) minimizers of the total energy $\mathcal{E}$, viewed as a functional defined on an appropriate set of admissible functions. In this section, we show that minimization of the energy functional $\mathcal{E}$ given by (3.10) is well-posed, that is: an energy minimizer exists for each rod homotopy class and one has an appropriate continuous dependence on data. We treat these two aspects of well-posedness separately.

Theorem 4. Let the conditions $(\mathcal{H})$ of Lemma 4 hold along with (3.5) and (3.6); suppose some admissible triple $\left[\mathrm{U}_{0}, \mathbf{q}_{0}, \mathbf{r}_{0}\right]$ is specified with finite energy. Then $\mathcal{E}$ attains its minimum over the class of admissible triples rod homotopic to $\left[\mathrm{U}_{0}, \mathbf{q}_{0}, \mathbf{r}_{0}\right]$.

Proof. Let $\left[\mathrm{U}_{k}, \mathbf{q}_{k}, \mathbf{r}_{k}\right]$ be a minimizing sequence for $\mathcal{E}$ in this class of admissible triples, hence bounded in total energy and rod homotopic to each other. As a bound on $\mathcal{E}$ implies a bound on the $L^{2}$-norms $\left\|\mathrm{U}_{k}\right\|$, we can extract a weakly convergent subsequence $\mathrm{U}_{k} \rightarrow \overline{\mathrm{U}}$; using Lemma 2 we see that this bound on $\left\|\mathrm{U}_{k}\right\|$ also implies a uniform positive lower bound on the margins of separation so we have $\left[\mathrm{U}_{k}, \mathbf{q}_{k}, \mathbf{r}\right] \rightarrow$ $[\overline{\mathbf{U}}, \overline{\mathbf{q}}, \overline{\mathbf{r}}]$. By Corollary 2 to Theorem 2 , the limit $[\overline{\mathbf{U}}, \overline{\mathbf{q}}, \overline{\mathbf{r}}]$ is admissible and in the same homotopy class - indeed, in the same strong rod homotopy class if $\left(\mathcal{H}^{\prime}\right)$ is assumed. The lower semicontinuity assumed in $(\mathcal{H})$ ensures that the energy minimum is then attained there.

There is no reason to expect, in general, that the minimizer for each rod homotopy class is unique, so we cannot expect continuous dependence on the constitutive functions. What is appropriate here is that, given $[\mathcal{W}, \mathcal{F}, \mathcal{G}]$, each of these sets of minimizers is closed and the set is upper semicontinuous in its dependence on the functional $\mathcal{E}$ of (3.10). To consider this dependence, we define convergence of a sequence of energy functionals $\mathcal{E}_{k}[\cdot]$ as follows:

Definition 5. A sequence of energy functionals $\mathcal{E}_{k}$ converges to $\mathcal{E}_{\infty}$ if

a. the coercivity condition (3.9) on $\mathcal{W}_{k}$ is uniform in $k$ 
b. for each fixed $\mathrm{U}$ such that $\mathcal{E}_{\infty}[\mathrm{U}]<\infty$, we have $\mathcal{E}_{k}[\mathrm{U}] \rightarrow \mathcal{E}_{\infty}[\mathrm{U}]$ in $\overline{\mathbb{R}}$

c. for each $M$ there is a sequence $\varepsilon_{k}^{M} \rightarrow 0$ such that $\mathcal{E}_{\infty}[\mathrm{U}] \leq \mathcal{E}_{k}[\mathrm{U}]+\varepsilon_{k}^{M}$ whenever $\mathcal{E}_{\infty}[\mathrm{U}] \leq M$.

Theorem 5. Suppose we are given $\mathrm{U}_{0}$ with $\mathcal{E}_{\infty}\left[\mathrm{U}_{0}\right]$ finite and a sequence of energy functionals $\mathcal{E}_{k}$ converging to $\mathcal{E}_{\infty}$ as in Definition 5 above and let $\mathrm{U}_{k}$ be minimizers of $\mathcal{E}_{k}$ over the rod homotopy class of $\mathrm{U}_{0}$. Then (for a subsequence) one has $\mathrm{U}_{k} \rightarrow \mathrm{U}_{\infty}$ and this $\mathrm{U}_{\infty}$ minimizes $\mathcal{E}_{\infty}$ over the same rod homotopy class.

Proof. Since $\mathcal{E}_{k}\left[\mathrm{U}_{0}\right] \rightarrow \mathcal{E}_{\infty}\left[\mathrm{U}_{0}\right]<\infty$, we have each $\mathcal{E}_{k}\left[\mathrm{U}_{0}\right]$ finite (for large enough $k$ ) so, by Theorem 4 , the minimizers $\bigcup_{k}$ exist. Further, we have $\left\{\mathcal{E}_{k}\left[\mathrm{U}_{0}\right]\right\}$ bounded so, by the assumed uniform coercivity of $\mathcal{W}_{k}$, we have $\left\{\left\|\mathrm{U}_{k}\right\|\right\}$ bounded whence (for a subsequence) we have $\mathrm{U}_{k} \rightarrow \mathrm{U}_{\infty}$ for some $\mathrm{U}_{\infty}$. As each $\mathrm{U}_{k}$ is admissible and in the rod homotopy class of $U_{0}$, so is $U_{\infty}$ by Corollary 2. Further, Theorem 4 gives existence of such a minimizer $\bar{U}$ for $\mathcal{E}_{\infty}$. We need only show that the limit $U_{\infty}$ is itself such a minimizer, i.e., that $\mathcal{E}_{\infty}\left[U_{\infty}\right]=\mathcal{E}_{\infty}[\bar{U}]=\min \left\{\mathcal{E}_{\infty}\right\}$.

Note that lower semicontinuity of $\mathcal{E}_{\infty}$ gives $\mathcal{E}_{\infty}\left[\mathrm{U}_{\infty}\right] \leq \mathcal{E}_{\infty}\left[\mathrm{U}_{k}\right]+\tilde{\varepsilon}_{k}$ with $\tilde{\varepsilon}_{k} \rightarrow 0$. Thus, we have $\mathcal{E}_{\infty}\left[\mathrm{U}_{k}\right] \leq \mathcal{E}_{k}\left[\mathrm{U}_{k}\right]+\varepsilon_{k}^{M}$ so $\min \left\{\mathcal{E}_{\infty}\right\} \leq \mathcal{E}_{\infty}\left[\mathrm{U}_{\infty}\right] \leq \liminf \min _{k}\left\{\mathcal{E}_{k}\right\}$. On the other hand, we have $\left.\min \left\{\mathcal{E}_{k}\right\} \leq \mathcal{E}_{k}[\overline{\mathrm{U}}]\right\}$ for each $k$ and $\left.\mathcal{E}_{k}[\overline{\mathrm{U}}]\right\} \rightarrow \mathcal{E}_{\infty}[\overline{\mathrm{U}}]$ so $\liminf \operatorname{inin}_{k}\left\{\mathcal{E}_{k}\right\} \leq \mathcal{E}_{\infty}[\overline{\mathrm{U}}]=\min \left\{\mathcal{E}_{\infty}\right\}$.

\section{Optimality Conditions and Regularity}

At a local minimum, $[U, \mathbf{q}=\mathbf{Q}(\mathrm{U}), \mathbf{r}=\mathbf{R}(\mathbf{q})]$ is certainly an admissible triple with the inherent regularity given by Definition 1 . In view of the clearance inequality implied by Lemma 3, this is interior to the admissible triples. Thus, assuming some differentiability, we expect $\mathcal{E}^{\prime}[U]=0$ at the minimum as a necessary condition for optimality. Given suitable smoothness $\left(\mathbf{H}^{\prime \prime}\right)$ of the constitutive functions, we can then interpret this necessary condition as an "Euler equation" for the optimizer and use that to show additional regularity for $\mathrm{U}(\cdot)$. 
$\left(\mathbf{H}^{\prime \prime}\right)$

a. The constitutive function $W:[0,1] \times \mathbb{R}^{3} \longrightarrow\left[-\beta_{W}, \infty\right)$ is convex and satisfies

$$
a|\mathrm{U}|-b \leq W(s, \mathrm{U}) \leq \hat{a}|\mathrm{U}|+b \quad(a, \hat{a}>0) .
$$

b. $W$ is differentiable in $\mathrm{U}$ with $W_{\mathrm{U}}$ injective in $\mathrm{U}$ for each $s$; $W_{\mathrm{U}}:[0,1] \times \mathbb{R}^{3} \longrightarrow \mathbb{R}^{3}$ is continuous.

c. Where finite, $G:[0,1]^{2} \times \mathbb{R}_{+} \longrightarrow\left[-\beta_{G}, \infty\right]$ is differentiable in $D$ with $G_{D}$ continuous in $(s, \sigma, D)$.

d. $F:[0,1] \times \mathbb{R}^{3} \longrightarrow \mathbb{R}$ is differentiable in $\mathbf{r}$ with $F_{\mathbf{r}}$ continuous in $(s, \mathbf{r})$.

Lemma 5. Assume the strengthened hypotheses $\left(\mathbf{H}^{\prime \prime}\right)$ and let $[\mathrm{U}, \mathbf{q}, \mathbf{r}]$ be any triple for which $\mathcal{E}[\mathrm{U}]$ is finite with $\mathbf{r}=\mathbf{R}(\mathbf{q}), \mathbf{q}=\mathbf{Q}(\mathrm{U})$. Then $\mathcal{W}[\mathrm{U}], \mathcal{G}[\mathbf{r}], \mathcal{F}[\mathbf{r}]$ and $\mathcal{E}[\mathrm{U}]$ are Fréchet differentiable there with

$$
\begin{aligned}
& \frac{d \mathcal{W}[\mathbf{U}]}{d \mathbf{U}}(s)=W_{\mathrm{U}}(s, \mathrm{U}(s)), \\
& \frac{d \mathcal{G}[\mathbf{r}]}{d \mathbf{r}}(s)=\int_{0}^{1} \frac{G_{D}(s, \sigma, D)+G_{D}(\sigma, s, D)}{D(s, \sigma)} \mathbf{r}(\sigma) d \sigma \\
& \text { with } D=D(s, \sigma)=|\mathbf{r}(s)-\mathbf{r}(\sigma)|, \\
& \frac{d \mathcal{F}[\mathbf{r}]}{d \mathbf{r}}(s)=F_{\mathbf{r}}(s, \mathbf{r}(s)) .
\end{aligned}
$$

and

$$
\begin{aligned}
\frac{d \mathcal{E}[\mathrm{U}]}{d \mathbf{U}}(s) & =W_{\mathbf{U}}(s, \mathrm{U}(s))+S^{* F}\left[\mathbf{q}^{\top}(s) \Theta(s)\right] \quad \text { where } \\
-\Theta^{\prime} & =[R(S(\mathbf{U}(s)))]^{* F} \Theta+\left(0,0, \int_{s}^{1} v(\hat{s}) d \hat{s}\right) \quad \Theta(1)=\mathbf{0} \\
v(s) & =[d \mathcal{G}[\mathbf{r}] / d \mathbf{r}](s)+[d \mathcal{F}[\mathbf{r}] / d \mathbf{r}](s) \quad \text { using }(5.2),(5.3) .
\end{aligned}
$$

Proof. Given any $\mathfrak{u} \in L^{2}\left([0,1] \rightarrow \mathbb{R}^{3}\right)$ we have $\mathcal{W}[\mathrm{U}+\tau \mathfrak{u}]<\infty$ for each $\tau$ by a. of $\left(\mathbf{H}^{\prime \prime}\right)$ and convexity of $W$ gives convexity of $\tau \mapsto W(s, \mathrm{U}(s)+\tau \mathfrak{u}(s))$ for each $s$. Thus, for $0<\tau \leq 1$ one has

$$
W[\mathbf{U}-\mathfrak{u}]-W[\mathbf{U}] \leq \frac{W[\mathbf{U}+\tau \mathfrak{u}]-W[\mathbf{U}]}{\tau} \leq W[\mathbf{U}+\mathfrak{u}]-W[\mathbf{U}]
$$


pointwise in $s$. We then apply Lebesgue's Dominated Convergence Theorem to get

$$
\begin{aligned}
\lim _{\tau \rightarrow 0+} \frac{\mathcal{W}[\mathrm{U}+\tau \mathfrak{u}]-\mathcal{W}[\mathrm{U}]}{\tau} & =\int_{0}^{1} \lim _{\tau \rightarrow 0+} \frac{W(s, \mathrm{U}(s)+\tau \mathfrak{u}(s))-W(s, \mathrm{U}(s))}{\tau} d s \\
& =\int_{0}^{1} W_{\mathfrak{U}}(s, \mathrm{U}(s)) \cdot \mathfrak{u}(s) d s \quad=\left\langle W_{\mathbf{U}}, \mathfrak{u}\right\rangle .
\end{aligned}
$$

Since this holds for each $\mathfrak{u}$, we have Gâteaux differntiability in every direction and (5.1); since $\mathrm{U}(\cdot) \mapsto \mathcal{W}^{\prime}[\mathrm{U}]=W_{\mathrm{U}}(\cdot, \mathrm{U}(\cdot))$ is continuous from $L^{2}\left([0,1] \rightarrow \mathbb{R}^{3}\right)$ to itself by Krasnosel'skii's theorem on Nemytskii operators, it follows that this is actually a Fréchet derivative.

The arguments for (5.2), (5.3) are even simpler since $\mathbf{r}$ is continuous, hence with compact range, and similarly the directions $\mathfrak{r}$ are continuous. The formula (5.3) is immediate; note that, since $\mathbf{r}$ is continuous, $v_{F}=\mathcal{F}^{\prime}[\mathbf{r}]$ given by (5.3) is continuous. For $\mathcal{G}^{\prime}$ we similarly get

$$
\lim _{\tau \rightarrow 0+} \frac{\mathcal{G}[\mathbf{r}+\tau \mathfrak{r}]-\mathcal{G}[\mathbf{r}]}{\tau}=\int_{0}^{1} \int_{0}^{1} G_{D}(s, \sigma, D) \frac{\mathfrak{r}(s)-\mathfrak{r}(\sigma)}{D} d s d \sigma,
$$

which, with a bit of manipulation, becomes (5.2); again we see from (5.2) that the function $v_{G}=\mathcal{G}^{\prime}[\mathbf{r}]$ is continuous. [We have an apparent possibility of singularity in having $D$ in the denominator, but recall Lemma 3.] By continuity, these are actually Fréchet derivatives.

Since, for each $\mathfrak{u}$, we have $\left\langle\mathcal{E}^{\prime}, \mathfrak{u}\right\rangle=\left\langle\mathcal{W}^{\prime}, \mathfrak{u}\right\rangle+\left\langle\mathcal{G}^{\prime}+\mathcal{F}^{\prime}, \mathfrak{r}\right\rangle$ with $\mathfrak{r}=\mathbf{L} \mathfrak{u}$, application of Lemma 1 gives the system (5.4).

Theorem 6. Assume the hypotheses $\left(\mathbf{H}^{\prime \prime}\right)$ and let $\mathbf{U}$ be a local minimizer of the energy $\mathcal{E}$. Then $\mathrm{U}(\cdot)$ is a continuous solution of the optimality system:

$$
W_{\mathrm{U}}(s, \mathrm{U}(s))=w(s)
$$

with

$$
\begin{aligned}
w(s) & =-S^{* F}\left[\mathbf{q}^{\boldsymbol{\top}}(s) \Theta(s)\right] \quad \text { where } \\
-\Theta^{\prime} & =[R(S(\mathbf{U}(s)))]^{* F} \Theta+\left(0,0, \int_{s}^{1} v(\hat{s}) d \hat{s}\right) \quad \Theta(1)=\mathbf{0} \\
v(s) & =[d \mathcal{G}[\mathbf{r}] / d \mathbf{r}](s)+[d \mathcal{F}[\mathbf{r}] / d \mathbf{r}](s) \quad \text { using }(5.2),(5.3) .
\end{aligned}
$$

One can obtain still further regularity of $\mathrm{U}(\cdot)$ to a level limited only by the assumed regularity of the constitutive functions if $W_{\mathrm{UU}}$ is an invertible matrix at each point: For $k=1, \ldots$, if $G_{D}, F_{\mathbf{r}}$ are in $C^{k-2}$ and $W_{\mathrm{U}}$ is in $C^{k}$ in a neighborhood of the rod, then one has $\mathrm{U}(\cdot), \mathbf{q}(\cdot), \mathbf{r}(\cdot)$ in $C^{k}, C^{k+1}, C^{k+2}$, respectively. 
Proof. Local optimality of $\boldsymbol{U}$ requires $\left\langle\mathcal{E}^{\prime}, \mathfrak{u}\right\rangle \geq 0$ for feasible directions $\mathfrak{u}$. Since we are considering only soft contact, it follows from Lemma 5 that all directions are feasible; in particular, positivity both with $\mathfrak{u}$ and $-\mathfrak{u}$ gives $\left\langle\mathcal{E}^{\prime}, \mathfrak{u}\right\rangle=0$ which means (5.5), (5.6) using (5.4) from Lemma 5. [Even when $\mathcal{W}$ is not differentiable, convexity would give

$$
-\mathbf{L}^{*}\left(\mathcal{G}^{\prime}[\mathbf{r}]+\mathcal{F}^{\prime}[\mathbf{r}]\right) \in \partial \mathcal{W}[\mathrm{U}]
$$

where the subgradient $\partial \mathcal{W}[\mathrm{U}]$ is given by the set of support functionals at $(\mathrm{U}, \mathcal{W}[\mathrm{U}])$ to the convex epigraph.]

Now look at the identity (5.5) on $[0,1]$. We know that $v=\mathcal{G}^{\prime}[\mathbf{r}]+\mathcal{F}^{\prime}[\mathbf{r}]$ is at least in $L^{2}$ so (5.6) gives $\Theta$, hence also $w$, in $H^{1}$ and so continuous. [The right hand side of the differential equation for $\Theta$ would be at least in $H^{1}$, giving $\Theta$ in $H^{2}$, if $\mathrm{U}$ were in $H^{1}$, but we do not know that at this time.]

Continuity of $w(\cdot)$ on $[0,1]$ implies a uniform bound on $w(s)$ whence, by (a) of $\left(\mathbf{H}^{\prime \prime}\right)$, a bound $\beta$ such that $\mathrm{U}(s) \in B_{\beta}=\left\{\mathrm{v} \in \mathbb{R}^{3}:|\mathrm{v}| \leq \beta\right\}$. Noting that $[0,1] \times B_{\beta}$ is compact, we consider the function

$$
\Psi:(s, \mathrm{v}) \longmapsto\left(s, W_{\mathrm{U}}(s, \mathrm{v})\right):[0,1] \times B_{\beta} \longrightarrow[0,1] \times \mathbb{R}^{3}
$$

By a. of $\left(\mathbf{H}^{\prime \prime}\right)$, this is injective, hence a bijection to its (necessarily compact) range and so has a continuous inverse. Composing $\Psi^{-1}$ with the continuous function $w(\cdot)$ then shows that $s \mapsto \mathrm{U}(s)$ is continuous, as asserted.

Now suppose each $W_{\mathrm{UU}}$ is invertible. Then, by the Implicit Function Theorem, if $W_{\mathrm{U}}(\cdot, \cdot)$ has $C^{k}$ regularity, then $w(\cdot)$ in $(5.5)$ and $\mathrm{U}(\cdot)$ will each have the same regularity up to that level. We can bootstrap this regularity, proceeding by induction.

Suppose, then, that we know $\mathrm{U}(\cdot)$ is in $C^{k-1}$ so the coefficient $[R(S(\mathrm{U}(\cdot)))]^{* F}$ of (5.4) is in $C^{k-1}$ so (2.6) gives $\mathbf{q}=\mathbf{Q}(\mathbf{U})$ in $C^{k}$ and then $\mathbf{r}=\mathbf{R}(\mathbf{q})$ in $C^{k+1}$. For $G_{D}, F_{\mathbf{r}}$ in $C^{k-2}$, this certainly gives $v(\cdot)$ in $C^{k-2}$ by (5.2) and (5.3) and $\left(0,0, \int v\right)$ in $C^{k-1}$. The right hand side of the $\Theta$ equation in (5.6) will then be in $C^{k-1}$ giving $\Theta$ as well as $\mathbf{q}$ in $C^{k}$. Hence $w$ and $\mathbf{U}$ are then in $C^{k}$.

\section{Further Coupling}

Our existence proof depended heavily on the assumption that $\mathcal{W}$ is convex, but in some situations it is of interest to modify that. The dependence on $s$ of the constitutive function $W(s, \cdot)$ was intended to allow for variation of material properties along the rod. We have been taking these as intrinsic, but one might imagine temperaturedependent properties - $W=W(s, T(s), \mathrm{U}(s))$ - with the temperature $T(s)$ given, e.g., by

$$
-\left(a(s) T_{s}\right)_{s}=\psi(T, \mathbf{r}), \quad T_{s}(0)=0=T_{s}(1)
$$


It is natural for the source term $\psi$ to depend on the position $\mathbf{r}(s)$. One may still think of $\mathcal{W}=\int W$ as a functional $\mathcal{W}[\mathrm{U}]$, since $T(\cdot)$ depends on $\mathrm{U}$, but, unfortunately, we cannot now expect this new functional to be convex so we write the functional as $\hat{\mathcal{W}}[\mathrm{U}, T]$, separating the dependences. Nevertheless, it remains plausible to retain convexity for the dependence of $W$ on $\mathrm{U}(s) \in \mathbb{R}^{3}$ and the argument for an existence theorem in this setting will then be a cross between those for Theorems 4 and 5 .

We now impose the following assumptions on the constitutive functions $W, F, G$.

a. Each of the constitutive functions $G, F$ is lower semicontinuous in its variables; $W$ is lower semicontinuous in $s, \mathrm{U}$ and Lipschitzian in $T$ :

$$
|W(s, T, \mathrm{U})-W(s, \tilde{T}, \mathrm{U})| \leq L|T-\tilde{T}|
$$

with $L=L(\mathrm{U}) \leq C\left(1+|\mathrm{U}|^{2}\right)$.

b. $W:[0,1] \times \mathbb{R} \times \mathbb{R}^{3} \longrightarrow\left[-\beta_{W}, \infty\right]$ is convex in its third variable and satisfies (3.9) uniformly in $s, T$.

c. $G:[0,1]^{2} \times \mathbb{R}_{+} \longrightarrow\left[-\beta_{G}, \infty\right]$ satisfies (3.5) and (3.6).

d. $F:[0,1] \times \mathbb{R}^{3} \longrightarrow \mathbb{R}$ is bounded below on bounded sets.

e. $a(\cdot)>0$ and $\psi$ satisfy conditions ensuring that (6.1) defines a continuous map: $\mathbf{r}(\cdot) \mapsto T(\cdot) \in C([0,1])$.

Theorem 7. Let the functional $\mathcal{E}[\mathrm{U}]$ be given as in (3.10) except that one now has $W=W(s, T(s), \mathrm{U}(s))$ in the integrand defining the local internal energy $\hat{\mathcal{W}}$. We write

$$
\mathcal{E}[\mathrm{U}]=\hat{\mathcal{W}}[\mathrm{U}, T]+\mathcal{G}[\mathbf{r}]+\mathcal{F}[\mathbf{r}] \quad \hat{\mathcal{W}}[\mathrm{U}, T]=\int_{0}^{1} W(s, T(s), \mathrm{U}(s)) d s
$$

and the minimization over $\mathrm{U}$ is to be coupled with (6.1) as a constraint. Assume the conditions $(\hat{\mathbf{H}})$ and suppose some admissible triple $\left[\mathrm{U}_{0}, \mathbf{q}_{0}, \mathbf{r}_{0}\right]$ is specified with finite energy. Then $\mathcal{E}$ attains its minimum over the class of admissible triples rod homotopic to $\left[\mathrm{U}_{0}, \mathbf{q}_{0}, \mathbf{r}_{0}\right]$.

Proof. As usual, we begin by letting $\left[\mathrm{U}_{k}, \mathbf{q}_{k}, \mathbf{r}_{k}\right]$ be a minimizing sequence for $\mathcal{E}$ in this class of admissible triples, hence rod homotopic to each other and bounded in 
total energy; by the assumed uniform coercivity we may take $\left\{\mathrm{U}_{k}\right\}$ weakly convergent: $\mathrm{U}_{k} \rightarrow \mathbf{U}_{\infty}$. By Theorem 1 we then have $\mathbf{q}_{k}=\mathbf{Q}\left(\mathrm{U}_{k}\right) \rightarrow \mathbf{q}_{\infty}=\mathbf{Q}\left(\mathrm{U}_{\infty}\right)$ and similarly $\mathbf{r}_{k} \rightarrow \mathbf{r}_{\infty}$. The rod homotopy follows from Theorem 2 .

Using e. of $(\hat{\mathbf{H}})$, we also have uniform convergence $T_{k} \rightarrow T_{\infty}$. For $k=1, \ldots, \infty$, we define

$$
\mathcal{E}_{k}[\mathrm{U}]=\mathcal{W}_{k}[\mathrm{U}]+\mathcal{G}[\mathbf{r}]+\mathcal{F}[\mathbf{r}] \quad \text { with } \mathcal{W}_{k}[\mathrm{U}]=\hat{\mathcal{W}}\left[\mathrm{U}, T_{k}\right]
$$

- noting that $\mathcal{E}_{\infty}\left(\mathrm{U}_{\infty}\right)=\mathcal{E}\left[\mathrm{U}_{\infty}\right]$ and that $\mathcal{W}_{\infty}$ is convex so

$$
\liminf _{k} \mathcal{E}_{\infty}\left[\mathrm{U}_{k}\right] \geq \mathcal{E}_{\infty}\left[\mathrm{U}_{\infty}\right]=\mathcal{E}\left[\mathrm{U}_{\infty}\right] \geq \inf _{U} \mathcal{E}=\lim _{k} \mathcal{E}_{k}\left[\mathrm{U}_{k}\right]
$$

Now, using a. of $(\hat{\mathbf{H}})$, we have

$$
\begin{aligned}
\left|\mathcal{E}_{\infty}\left[\mathrm{U}_{k}\right]-\mathcal{E}_{k}\left[\mathrm{U}_{k}\right]\right| & =\left|\hat{\mathcal{W}}\left[\mathrm{U}_{k}, T_{\infty}\right]-\hat{\mathcal{W}}\left[\mathrm{U}_{k}, T_{k}\right]\right| \\
& \leq \int_{0}^{1} L\left(\mathrm{U}_{k}(s)\right)\left|T_{\infty}(s)-T_{k}(s)\right| d s \\
& \leq \int_{0}^{1} C\left(1+\left|\mathrm{U}_{k}(s)\right|^{2}\right) d s\left\|T_{\infty}-T_{k}\right\|_{\infty}
\end{aligned}
$$

Since $\left\{\mathrm{U}_{k}\right\}$ is $L^{2}$-bounded and $\left\|T_{\infty}-T_{k}\right\|_{\infty} \rightarrow 0$, the difference vanishes in the limit. Thus we must have equalities in (6.3) and $U_{\infty}$ is the desired minimizer.

[The same approach would apply for more general situations in which $\mathcal{W}$ depends also on $(\mathbf{q}, \mathbf{r})$, whether or not this dependence occurs through additional coupling.]

\section{Discussion}

The focus of this work has been to develop a framework for elastic rods with a pairwise repulsive potential which prevents non-adjacent points along the rod from occupying the same physical space. As in the classical Cosserat rod formulation, we assume only short range forces, but distinguish between local and nonlocal interactions. Within this framework, we have shown existence of an energy minimizer within each homotopy class, where the standard definition of homotopy has been suitably extended to include the frame of directors as well as the centerline of the rod. Under additional hypotheses, first-order necessary conditions were derived and used to obtain regularity.

The existence proof presented in section 4 is not constructive in the sense of determining the shape of the minimizing configuration of the elastic rod, beyond the 
regularity results discussed in section 5. For most elastic rod problems, including the ones presented here, analytic solutions are not attainable and numerical methods need to be developed to identify the minimal configurations. One method that has appeared in the literature is the use of Monte Carlo techniques to locate global minima, (see [38], for example). Another approach is to find families of critical points, using second-order information to identify which of these critical points correspond to minima, (see $[12,20]$, for example). Neither of these methods guarantees the existence of the minimizer, but each complements the present work by providing numerical approximations of the minimal configurations.

The formulation of the elastic rod energy presented here included only elastic potential energy, repulsive potential energy and external potential energy, but does not include, for instance, time dynamics and kinetic energy. Strict local minima of the static problem considered here correspond to stable stationary points of the dynamic equations. Our hypotheses, however, do not ensure uniqueness within each rod homotopy class or isolation of the minimizers, which is necessary for a stationary point to be stable for the time dependent dynamics. The second variation formulation presented in [20] may be used to identify minimizers with this property. In fact, the study of time-dependent stable stationary points has been addressed in the context of a bio-elastic filaments [22] and the binding of LacR protein by DNA and looping $[17,18]$.

We remark that the existence results presented here can be extended to include spatial obstacles, that is, regions $\mathcal{R}$ which the rod should not penetrate. Considerations essentially similar to Lemma 3 would apply to the external potential term $\mathcal{F}$ in this situation. When preventing self-intersection, we let $F(s, \mathbf{r})$ be such that $F(s, \mathbf{r})=\infty$ if $\mathbf{r} \in \mathcal{R}$ and, to ensure a positive margin for soft contact with the obstacle, we impose, as a condition on $F$, that $\hat{\gamma}(\cdot ; F)$ satisfies

$$
\int_{0+}^{a} \hat{\gamma}(r) d r=+\infty
$$

where imitating (3.4),

$$
\hat{\gamma}(r)=\inf \{F(s, \operatorname{dist}(\mathbf{r}, \mathcal{R})): \operatorname{dist}(\mathbf{r}, \mathcal{R}) \leq r\}
$$

An argument similar to the proof of Lemma 3 provides a clearance inequality preventing any contact with the obstacle: a bound on the external energy potential $\mathcal{F}[\mathbf{r}]$ implies a strictly positive lower bound on the clearance $[\operatorname{dist}(\mathbf{r}, \mathcal{R})]$. In view of this, we continue to have the results of Theorem 3 even though we have dropped the requirement that $F$ should be bounded. 


\section{Appendix}

\subsection{Proof of Theorem 2}

Proof. The details of the proof are complicated somewhat by the variety of alternative boundary conditions which might be imposed at $s=1$, each requiring a somewhat different treatment.

We begin by considering the special case in which $\mathbf{r}(1)=\mathbf{k}$ is specified. Since we have assumed an inextensible elastic rod, this boundary condition $\mathbf{r}(1)=\mathbf{k}$ implies $\mathbf{d}_{3}(s) \equiv \mathbf{k}$, and that $\mathbf{d}_{1}, \mathbf{d}_{2}$ lie in the $x-y$ plane, and hence have the form

$$
\overline{\mathbf{d}}_{1}(s)=\cos (\bar{\delta}(s)) \mathbf{i}+\sin (\bar{\delta}(s)) \mathbf{j}, \quad \overline{\mathbf{d}}_{2}(s)=-\sin (\bar{\delta}(s)) \mathbf{i}+\cos (\bar{\delta}(s)) \mathbf{k},
$$

with similar expressions for $\hat{\mathbf{d}}_{1}, \hat{\mathbf{d}}_{2}$ replacing $\bar{\delta}$ by $\hat{\delta}$. Since these triples are admissible, we have $\hat{\delta}, \bar{\delta} \in H^{1}(0,1)$ and define $\Psi(s)=\hat{\delta}(s)-\bar{\delta}(s)$. As $\overline{\mathbf{q}}(0)=\mathbf{I}=\hat{\mathbf{q}}(0)$ we may assume $\bar{\delta}(0)=0=\hat{\delta}(0)$ so $\Psi(0)=0$ and $\Psi$ will remain uniformly small for $\hat{\mathbf{q}}$ close to $\overline{\mathbf{q}}$. We define the rod homotopy by

$$
\mathbf{q}(s, t)=\left(\begin{array}{c}
\cos (\bar{\delta}+t \Psi) \mathbf{i}+\sin (\bar{\delta}+t \Psi) \mathbf{j}] \\
-\sin (\bar{\delta}+t \Psi) \mathbf{i}+\cos (\bar{\delta}+t \Psi) \mathbf{j}] \\
\mathbf{k}
\end{array}\right) .
$$

Since $\bar{\delta}, \hat{\delta} \in H^{1}(0,1)$, it follows that $\mathbf{q}(\cdot, t)$ is bounded in $H^{1}([0,1] \rightarrow S O(3))$ and condition $(d)$ is satisfied. The boundary conditions $(c)$ and the continuity $(b)$ with respect to $t$ are certainly satisfied by this construction. Since $D_{*}$ depends continuously on $\mathbf{r}$ and $\mathbf{r}(\cdot, t)$ stays close to $\overline{\mathbf{r}}$ since $\Psi$ stays small, we have $D_{*}(t)$ close to $D_{*}(0)>0$, we have $(e)$ for a small enough neighborhood $\mathcal{N}$. Finally, to verify $(a)$ we note that Theorem 1 ensures existence of a suitable $\mathrm{U}(\cdot, t)$ and for admissibility it only remains to check the condition $\mathbf{q}(1)=\mathbf{q}_{*}$ if this is imposed. Since $\Psi$ is small we can have $\overline{\mathbf{q}}(1)=\mathbf{q}_{*}=\hat{\mathbf{q}}$ only if $\Psi(1)=0$, which then gives $\mathbf{q}(1, t) \equiv \mathbf{q}_{*}$ for each $t$.

For the remaining cases, in which either no condition is imposed on $\mathbf{r}(1)$ or we are imposing $\mathbf{r}(1)=\mathbf{r}_{*}$ with $\mathbf{r}_{*} \neq \mathbf{k}$, we will use a somewhat different construction of the rod homotopy. We begin by noting that $S O(3)$ is a Lie group so there is a $C^{\infty}$ diffeomorphism $\phi$ from the open unit ball $B$ of $\mathbb{R}^{3}$ to $S O(3)$ with $\phi(0)=\mathbf{I}$, the $3 \times 3$ identity matrix. Thus $\mathcal{U}=\phi(B)$ is a neighborhood of $\mathbf{I}$ in $S O(3)$ and, as a first requirement on the neighborhood $\mathcal{N}$, we ask that $\hat{\mathbf{q}} \in \mathcal{N}$ should imply $\left[\overline{\mathbf{q}}(s)^{-1} \hat{\mathbf{q}}(s)\right] \in \mathcal{U}$ for each $s \in[0,1]$ so we can define $\omega(\cdot):[0,1] \rightarrow B$ giving

$$
\left.\phi(\omega(s)) \equiv \overline{\mathbf{q}}^{-1}(s) \hat{\mathbf{q}}(s)\right) .
$$


Note that admissibility of $\hat{\mathbf{q}}, \overline{\mathbf{q}}$ gives $\hat{\omega}(\cdot) \in H^{1}([0,1] \rightarrow B)$ and that taking $\mathcal{N}$ small will ensure that $\omega$ is uniformly small. Our rod homotopy is to have the form

$$
\mathbf{q}(s, t)=\overline{\mathbf{q}}(s) \phi(t \omega(s)+M(s) \mathbf{a}(t))
$$

with $M:[0,1] \rightarrow \mathcal{M}^{3 \times 3}$ and $\mathbf{a}:[0,1] \rightarrow \mathbb{R}^{3}$ yet to be specified in such a way as to ensure the homotopy conditions. Note that the definition of $\phi$ ensures that (8.3) gives $\mathbf{q}(s, t) \in S O(3)$.

We note that when no condition is imposed on $\mathbf{r}(1)$ we may take $M \equiv \mathbf{0}$ (so a is irrelevant) and are then already assured of the desired conclusion. To see this, note that (8.3) then immediately gives $(b),(c)$, and $(d)$ by the smoothness of $\phi$ and $\phi^{-1}$ along with the given $H^{1}$ regularity of $\overline{\mathbf{q}}, \hat{\mathbf{q}}$. For small $\mathcal{N}$ we have $\mathbf{q}(\cdot, t)$ always close to $\overline{\mathbf{q}}$ which, as earlier, is sufficient to ensure $(e)$ so again it only remains to check the condition $\mathbf{q}(1)=\mathbf{q}_{*}$ if this is imposed. If so, then we have $\hat{\mathbf{q}}(1)=\overline{\mathbf{q}}(1)$ so $\omega(1)=0$ and the boundary condition for $\mathbf{q}$ is verified for all $t$.

For the final cases in which we are imposing $\mathbf{r}(1)=\mathbf{r}_{*}$ with $\mathbf{r}_{*} \neq \mathbf{k}$, we will cleverly construct a smooth matrix-valued function $M$, depending only on the given $\overline{\mathbf{q}}$. The form $(8.3)$ then defines $\mathbf{q}(\cdot, t)$ in $H^{1}([0,1] \rightarrow S O(3)$ with a uniform bound so long as a remains bounded and small enough to keep $[t \omega(s)+M(s) \mathbf{a}(t)$ in $\mathcal{U}$ then (b) is satisfied and we can obtain $\mathbf{U}, \mathbf{r}$ as above so $(d)$ will be satisfied. If a is kept smaller, then $\mathbf{q}(\cdot, t)$ stays close enough to $\overline{\mathbf{q}}$ that $(e)$ is satisfied. If we will have $\mathbf{a}(0)=\mathbf{a}(1)=0$, then $(c)$ is satisfied. If we require $M(1)=\mathbf{0}$, then, as above, any boundary condition $\mathbf{q}(1)=\mathbf{q}_{*}$ would be maintained. It is only necessary to show that the imposed condition $\mathbf{r}(1)=\mathbf{r}_{*}$ is maintained to verify $(a)$ and our construction of $M(\cdot)$ is directed to this end, enabling us to obtain $\mathbf{a}(t)$ so as to ensure

$$
\begin{gathered}
\Phi_{*}(t \omega, \mathbf{a}(t))=0 \\
\text { where } \quad \Phi_{*}(\hat{\omega}, \mathbf{a})=\int_{0}^{1}[\overline{\mathbf{q}}(s) \phi(\hat{\omega}(s)+M(s) \mathbf{a})]_{3} d t-\mathbf{r}_{*}
\end{gathered}
$$

Here $[\cdot]_{3}$ indicates extracting the third column of the matrix, which will just be $\mathbf{d}_{3}(s, t)$ so $\Phi_{*}(t \omega, \mathbf{a}(t))=[\mathbf{R}(\mathbf{q}(\cdot, t))](1)-\mathbf{r}_{*}=\mathbf{r}(1, t)-\mathbf{r}_{*}$. Note that $\Phi_{*}(0,0)=0$ since $\phi(0)=\mathbf{I}$ and $\overline{\mathbf{r}}$ satisfies the boundary condition at $s=1$. It is not hard to verify differentiability of $\Phi_{*}$ with

$$
\frac{\partial \Phi_{*}}{\partial \mathbf{a}}(0,0) \boldsymbol{\alpha}=\int_{0}^{1}\left[\overline{\mathbf{q}}(s) \phi^{\prime}(0) M(s) \boldsymbol{\alpha}\right]_{3} d s
$$

If we can construct $M$ so the $3 \times 3$ matrix $\partial \Phi_{*} / \partial \mathbf{a}(0,0)$ is invertible, then the Implicit Function Theorem (IFT) will ensure existence of a neighborhood $\mathcal{N}^{\prime}$ of 0 in 
$C\left([0,1] \rightarrow \mathbb{R}^{3}\right)$ and a unique continuous function $A: \hat{\omega} \mapsto \mathbf{a}$ giving $\Phi_{*}(\hat{\omega}, A(\hat{\omega})) \equiv 0$. Thus, for small enough $\mathcal{N}$, we will have each $t \omega$ in $\mathcal{N}^{\prime}$ and $\mathbf{a}(t)=A(t \omega)$ remaining small enough. The IFT gives $A(0)=0$ so $\mathbf{a}(0)=0$; for $t=1$ we have $\hat{\mathbf{r}}(1)=\mathbf{r}_{*}$ by assumption so $\Phi_{*}(\omega, 0)=0$ whence, by uniqueness, $A(\omega)=0$ so a $(1)=0$. With $M(1)=0$, that will complete our verification of $(a)$ and so complete the proof.

Our construction of $M(\cdot)$ begins with the observation that with $\overline{\mathbf{r}}(1)=\mathbf{r}_{*} \neq \mathbf{k}$ we cannot have both $\overline{\mathbf{d}}_{1}(s) \cdot \mathbf{k} \equiv 0$ and also $\overline{\mathbf{d}}_{2}(s) \cdot \mathbf{k} \equiv 0$. Hence we can assume, without loss of generality, that there is some $s_{*} \in(0,1)$ such that $\overline{\mathbf{d}}_{1}\left(s_{*}\right) \cdot \mathbf{k} \not \equiv 0$. Thus, we have

$$
\overline{\mathbf{d}}_{1}(0)=\mathbf{i}, \quad \overline{\mathbf{d}}_{2}(0)=\mathbf{j}, \quad \overline{\mathbf{d}}_{1}\left(s_{*}\right)=\delta_{1} \mathbf{i}+\delta_{2} \mathbf{j}+\delta_{3} \mathbf{k} \text { with } \delta_{3} \neq 0 .
$$

Next we note that the range of $\phi^{\prime}(0): \mathbb{R}^{3} \mapsto M^{3 \times 3}$ is the tangent space at $\mathbf{I}=\phi(0)$ to $S O(3)$, which is precisely the space of skew-symmetric $3 \times 3$ matrices, so there are $\mathbf{u}, \mathbf{v}$ such that

$$
\left[\phi^{\prime}(0) \mathbf{u}\right] \mathbf{k}=\mathbf{i} \quad\left[\phi^{\prime}(0) \mathbf{v}\right] \mathbf{k}=\mathbf{j}
$$

Taking $\mathbf{m}_{j}(s)=u_{j}(s) \mathbf{u}+v_{j}(s) \mathbf{v}$ as the columns of $M$, we note that the integrand in (8.5) becomes

$$
\begin{gathered}
{\left[\overline{\mathbf{q}}(s) \phi^{\prime}(0) M(s) \boldsymbol{\alpha}\right] \mathbf{k}=\sum_{j=1}^{3} \alpha_{j} \mathbf{c}_{j}(s)} \\
\text { with } \quad \mathbf{c}_{j}(s)=\overline{\mathbf{q}}(s)\left[\phi^{\prime}(0) \mathbf{m}_{j}(s)\right] \mathbf{k}=u_{j}(s) \overline{\mathbf{d}}_{1}(s)+v_{j}(s) \overline{\mathbf{d}}_{2}(s)
\end{gathered}
$$

To have invertibility of $\partial \Phi_{*} / \partial \mathbf{a}(0,0)$ we need $\left\{\int \mathbf{c}_{j}(s) d s\right\}$ independent; to ensure that, we find $u_{j}(s), v_{j}(s)$ so these integrals are approximately $\mathbf{i}, \mathbf{j}, \mathbf{k}$ for $j=1,2,3$. Recalling (8.6) and the continuity of $\overline{\mathbf{q}}(\cdot)$, we see that this is possible by taking

i: $\quad u_{1}(\cdot)$ to be a unit spike near $s \approx 0$ with $v_{1} \equiv 0$

j: $\quad v_{2}(\cdot)$ to be a unit spike near $s \approx 0$ with $u_{2} \equiv 0$

k: $\quad u_{3}(\cdot)$ to be $\left[\left(\right.\right.$ a unit spike near $\left.\left.s \approx s_{*}\right)-\delta_{1} u_{1}(\cdot)\right] / \delta_{3}$ with $v_{3}(\cdot)=-\left(\delta_{2} / \delta_{3}\right) v_{2}(\cdot)$ where by a 'unit spike' we mean a smooth function with narrow support and integral 1 , vanishing at $s=1$.

As indicated earlier, this completes the proof.

\section{Acknowledgements}

The authors thank Profs. Robert Manning and Stuart Antman for helpful discussions and suggestions. 


\section{References}

[1] S.S. Antman. Equilibrium states of nonlinearly elastic rods. J. Math. Anal. Appl., 23:459-470, 1968.

[2] S.S. Antman. Existence of solutions of the equilibrium equations for nonlinearly elastic rings and arches. Indiana Univ. Math. J., 1970.

[3] S.S. Antman. Existence and nonuniqueness of axis-symetric equilibrium states of nonlinearly elastic shells. Arch. Rat. Mech. Anal., 40:329-372, 1971.

[4] S.S. Antman. Ordinary differential equations of nonlinear elasticity II: Existence and regularity theory for conservative boundary value problems. Arch. Rat. Mech. Anal., 61:353-393, 1976.

[5] S.S. Antman. Nonlinear Problems in Elasticity. Springer, 2 edition, 2005.

[6] J. M. Ball. Convexity conditions and existence theorems in nonlinear elasticity. Arch. Rational Mech. Anal., 63:337-403, 1977.

[7] J.R. Banavar, O. Gonzalez, J.H. Maddocks, and A. Maritan. Self-interactions of strands and sheets. J. Stat. Phys., 110:35-50, 2003.

[8] P.G. Ciarlet and J. Nečas. Injectivity and self-contact in nonlinear elasticity. Arch Rat. Mech. Anal., 97:171-188, 1987.

[9] B.D. Coleman and D. Swigon. Theory of supercoiled elastic rings with selfcontact and its application to DNA plasmids. J. Elasticity, 60:171-221, 2000.

[10] B.D. Coleman, D. Swigon, and I. Tobias. Elastic stability of DNA configurations: II. supercoiled plasmids with self-contact. Phys. Rev. E, 61:759-770, 2000 .

[11] J.J. Delrow, J.A. Gebe, and J.M. Schurr. Comparison of hard cylinder and screened Coulomb interaction in the modeling of supercoiled DNAs. Biophysics, 42:455-470, 1997.

[12] D. J. Dichmann, Y. W. Li, and J. H. Maddocks. Hamiltonian formulations and symmetries in rod mechanics. In J.P. Mesirov, K. Schulten, and D.W. Sumners, editors, Mathematical Approaches to Biomolecular Structure and Dynamics, volume 82, pages 71-113. Springer, New York, 1996. IMA Volumes in Mathematics and its Applications. 
[13] E. Emmrich and O. Weckner. On the well-posedness of the linear peridynamic model and its convengence toward the Navier equation of linear elasticity. Comm. Math. Sci., 2007.

[14] S. Fukuhara. A Fete of Topology, chapter Energy of a Knot. Academic Press, 1988.

[15] O. Gonzalez and J. H. Maddocks. Global curvature, thickness and the ideal shape of knots. PNAS, 96(9):4769-4773, 1999.

[16] O. Gonzalez, J.H. Maddocks, F. Schuricht, and H. von der Mosel. Global curvature and self-contact of nonlinearly elastic curves and rods. Calc. Var. Partial Diff. Eqns., 14:29-68, 2002.

[17] S. Goyal, N.C. Perkins, and C. L. Lee. Non-linear dynamic intertwining of rods with self-contact. Nonlinear Mechanics, 43:65-73, 2008.

[18] S. Goyal, N.C. Perkins, and C.L. Lee. Nonlinear dyanmics and loop formation in Kirchhoff rods with implications to the mechanics of DNA cables. J. Comp. Phys., 2005.

[19] D. Habeck and F. Schuricht. Contact between nonlinearly elastic bodies. Proc. Roy. Soc. Edinburgh A, 136:1239-1266, 2006.

[20] K.A. Hoffman and R.S. Manning. Stability of elastic rods with repulsive potentials. submitted, 2008.

[21] V. Katritch, J. Bednar, D. Michoud, R.G. Scharein, J.U. Dubochet, and A. Stasiak. Geometry and physics of knots. Nature, 384:142-145, 1996.

[22] I. Klapper. Biological applications of the dynamics of twisted elastic rods. J. Comp. Phys., 125:325-337, 1996.

[23] I.A. Kunin. Elastic Media with Microstructure, volume I/II. Springer, 1982/83.

[24] R.B. Kusner and J.M. Sullivan. Möbius-invariant knot energies. In Stasiak, Katritch, and Kauffman, editors, Ideal Knots, volume 19 of Ser. on Knots and Everything, pages 315-352. World Scientific, 1998.

[25] R.B Lehoucq and S.A. Silling. Force flux and the peridynamic stress tensor. J. Mech. and Physics of Solids, 184:1566-1577, 2007.

[26] J. O’Hara. Energy of a knot. Topology, 30:241-247, 1991. 
[27] D. Rogula. Nonlocal Theory of Material Media. Springer, 1982.

[28] F. Schuricht. Variational approach to contact problems in nonlinear elasticity. Calculus of Variations, 15:433-449, 2002.

[29] F. Schuricht. Nonlinear analysis and Applications to Physical Sciences, chapter Contact Problems in Nonlinear Elasticity. Modeling, Analysis, and Application, pages 91-133. Springer, 2004.

[30] F. Schuricht. A new mathematical foundation for contact interations in continuum physics. Arch. Rational. Mech. Anal., 185:495-551, 2007.

[31] F. Schuricht and H. von der Mosel. Euler-Lagrange equations for nonlinearly elastic rods with self-contact. Arch. Rational Mech. Anal., 168:35-82, 2003.

[32] S.A. Silling. Reformulation of elasticity theory for discontinuities and longrange forces. J. Mech and Physics of Solids, 48:175-209, 2000.

[33] J. Simon. Energy functions for polygonal knots. J Knot theory and its ramifications, 3:299-320, 1994.

[34] E.L. Starostin. Closed loops of a thin elastic rod and its symmetric shapes with self-contacts. In Proc. 16th IMACS World Congress, Lausanne Switzerland, 21-25 August 2000, 2000.

[35] E.L. Starostin. Symmetric equilibria of a thin elastic rod with self-contacts. Phil. Trans. Roy. Soc. A, 362:1317-1334, 2004.

[36] P. Strzelecki and H. von der Mosel. On rectifiable curves with $L^{p}$ bounds on global curvature: Self-avoidance, regularity, and minimizing knots. Math. Z., 2007.

[37] G. vander Heijden, S. Neukirch, V.G.A.Goss, and J.M.T. Thompson. Instability and self-contact phenomena in the writhing of clamped rods. Intl. J. Mech. Sciences, 45:161-196, 2003.

[38] A. Vologodskii and N. Cozzarelli. Modeling of long-range electrostatic interactions in DNA. Biopolymers, 35:289-296, 1995. 